\title{
INDICADORES DE DESEMPENHO OPERACIONAL E ECONÔMICO: UM ESTUDO EXPLORATÓRIO NO CONTEXTO DO AGRONEGÓCIO'1
}

\author{
Antônio André Cunha Callado 2 \\ Aldo Leonardo Cunha Callado ${ }^{3}$ \\ Márcio André Veras Machado 4
}

\begin{abstract}
Resumo: Tendo em vista a importância da medição de desempenho para a gestão empresarial, 0 presente estudo teve por objetivo elaborar e aplicar indicadores de desempenho para comparar organizações industriais rurais localizadas no Estado do Ceará. Para a realização deste trabalho, foram pesquisadas 158 unidades rurais processadoras de raspas de mandioca, localizadas em 9 sub-regiões do Estado do Ceará. Os indicadores de desempenho foram agrupados obedecendo às variáveis relacionadas ao processo operacional da atividade-fim, bem como às variáveis vinculadas às atividades comerciais.
\end{abstract}

Palavras-chave: Indicadores de desempenho. Agronegócio.

\section{INTRODUÇÃO}

Os indicadores de desempenho aplicados em empresas rurais possuem características comuns aos aplicados nas demais organizações, compostos, inclusive, pelos mesmos elementos. As organizações associativas rurais se caracterizam pela atuação na agricultura e na pecuária, embora possam se inserir na categoria das atividades agroindustriais por desenvolverem atividades agrícolas através do processamento de matérias-primas.

De acordo com Marion (2000), as empresas rurais são definidas como aquelas que exploram a capacidade produtiva do solo por meio do cultivo da terra, da criação de animais e da transformação de determinados produtos agrícolas. Essas empresas podem explorar três categorias diferentes de atividades: as atividades agrícolas, as atividades zootécnicas e as atividades agroindustriais.

Considerando uma abordagem metodológica para a identificação do tamanho de uma empresa rural, Valle (1985) sugere um conjunto de elementos que devem ser levados em consideração na avaliação:

a) a superfície territorial da empresa (constitui o elemento principal para a classificação da dimensão empresarial);

b) as culturas existentes (quando a atividade principal for a agricultura);

c) as criações de gado ou de outras espécies de animais;

d) o sistema da organização e a gestão aziendal;

\footnotetext{
${ }_{1}^{1}$ Artigo publicado nos anais do V Congresso Brasileiro de Administração Rural, Campinas, SP, agosto/ 2005.

2 Doutorando em Administração, UFPE, Recife, PE. E-mail: andrecallado @ yahoo.com.br

${ }^{3}$ Departamento de Finanças e Contabilidade, UFPB, João Pessoa, PB. E-mail: aldocallado@ yahoo.com.br

${ }^{4}$ Departamento de Administração, UFPB, João Pessoa, PB. E-mail: marciomachado@ hs24.com.br
} 
e) o capital investido em implementos, maquinário, estoques de materiais existentes no armazém;

f) a quantidade de mão-de-obra normalmente necessária às operações da empresa;

g) a quantidade média anual das produções vegetais e animais.

A definição da dimensão empresarial ótima é obtida considerando, como elementos variáveis, todos os fatores produtivos investidos e, como elemento fixo, a capacidade administrativa da empresa. A dimensão ótima, segundo Valle (1985), seria proporcionada pela quantidade de fatores produtivos que o empreendedor agrário pode combinar com a sua capacidade de dirigente para obter o máximo resultado líquido do empreendimento.

As empresas rurais geralmente adotam estratégias de diversificação de produtos para enfrentar dificuldades decorrentes de riscos associados às sazonalidades. Comentando sobre as particularidades da gestão financeira dentro de empresas rurais, Mattos (1999) afirma que o produto de maior importância deverá determinar o tamanho do ciclo operacional.

Em geral, a maior parte das atividades rurais se desenvolve de forma irregular durante 0 exercício e a administração enfrenta o desafio de atenuar ou remediar a irregularidade natural do curso dos trabalhos, intensificando outras atividades conexas (beneficiamento ou industrialização dos produtos colhidos) ou reparando as benfeitorias.

Segundo Marion; Santos; Segatti (2002), o principal papel do administrador rural é planejar, controlar, decidir e avaliar os resultados, visando à maximização dos lucros, à permanente motivação e ao bem-estar de seus recursos humanos.

A contabilidade presta serviços aos dirigentes ou administradores, não somente nos aspectos financeiros, mas também em questões de grande importância para formular, reformular ou avaliar 0 processo administrativo.

Para a empresa rural, assim como para indústrias, a contabilidade é uma ferramenta de apoio na gestão, um meio utilizado para cumprir melhor os fins produtivos e sociais da empresa.

Tendo em vista a importância da medição de desempenho para a gestão empresarial, 0 presente estudo tem por objetivo elaborar e aplicar indicadores de desempenho para comparar as unidades processadoras de mandioca localizadas nas diversas sub-regiões do Estado do Ceará.

Este trabalho discorre sobre o agronegócio e os indicadores de desempenho, relata os procedimentos metodológicos, contém a apresentação e análise dos resultados e, por fim, a conclusão.

\section{AGRONEGÓCIO}

Segundo dados do Ministério da Agricultura, Pecuária e Abastecimento, o agronegócio é responsável por $33 \%$ do Produto Interno Bruto (PIB), $42 \%$ das exportações totais e $37 \%$ dos empregos brasileiros. Ainda de acordo com esse Ministério, entre 1998 e 2003, a taxa de crescimento do PIB agropecuário foi de $4,67 \%$ ao ano, e, nos últimos anos, poucos países tiveram um crescimento tão expressivo no comércio internacional do agronegócio quanto o Brasil.

O Ministério da Agricultura destaca que o país dobrou o faturamento com as vendas externas de produtos agropecuários em dez anos e teve um crescimento superior a $200 \%$ no saldo comercial. Esses resultados levaram a Conferência das Nações Unidas para o Comércio e Desenvolvimento (UNCTAD) a prever que o país será o maior produtor mundial de alimentos da próxima década.

Conforme informações do Ministério da Agricultura, Pecuária e Abastecimento, o Brasil é um dos líderes mundiais na produção e exportação de vários produtos agropecuários. É o primeiro produtor e exportador de café, açúcar, álcool e sucos de frutas. Além disso, lidera o ranking das vendas 
externas de soja, carne bovina, carne de frango, tabaco, couro e calçados de couro. As projeções indicam que o país também será, em pouco tempo, o principal pólo mundial da produção de algodão e biocombustíveis feitos a partir da cana-de-açúcar e óleos vegetais. Milho, arroz, frutas frescas, cacau, castanhas, nozes, além de suínos e pescados, são destaques do agronegócio brasileiro, que emprega atualmente 17,7 milhões de trabalhadores somente no campo.

Mazzali (2000), comentando sobre as transformações econômicas ocorridas nos anos 80 e seus reflexos na dinâmica recente do agronegócio brasileiro, afirma que novos condicionantes redirecionaram o comportamento dos agentes envolvidos com a atividade agroindustrial.

Essas mudanças estão relacionadas ao desenvolvimento científico e tecnológico, que provocaram repercussões tanto no processo produtivo, quanto nas formas de organização da produção e da implantação de novas estratégias empresariais.

Megido; Xavier (1998) comentam sobre o processo de inserção do agronegócio brasileiro no contexto internacional como uma necessidade estrutural para sua competitividade dentro dos macrocenários dos mercados mundiais, destacando duas perspectivas principais:

- conscientizar a sociedade sobre o potencial de internacionalização de nosso agribusiness;

- agir como agente catalisador na estruturação de redes de informação sobre o sistema agroalimentar brasileiro e internacional.

Em termos gerais, a competitividade pode ser entendida como a capacidade sustentável de sobreviver e, de preferência, crescer nos mercados concorrentes ou em novos mercados. Abordando a competitividade sob o enfoque dos sistemas agroindustriais em decorrência da globalização, Jank e Nassar (2000) afirmam que ela pode estar divida em três blocos:

- capacidade produtiva/tecnológica $\Rightarrow$ relacionadas às vantagens de custos que são reflexos da produtividade dos fatores de produção e/ou logística;

- capacidade de inovação $\Rightarrow$ relacionadas aos investimentos públicos ou privados em ciência, tecnologia e formação de capital humano;

- capacidade de coordenação $\Rightarrow$ relacionadas à capacidade de receber, processar, difundir e utilizar informações para definir e viabilizar estratégias competitivas (inovação de produtos/processos, diferenciação e segmentação), efetuar controles e reagir a mudanças no meio ambiente.

Nas empresas rurais, assim como nas demais modalidades de organizações, a gestão administrativa abrange dois aspectos principais: o processo operacional relacionado à atividade-fim $e$ as atividades comerciais. Os aspectos sobre o processo operacional se desenvolvem no âmbito da empresa, enquanto os aspectos sobre as atividades comerciais se desenvolvem entre as empresas e 0 ambiente externo.

As atividades que podem ser consideradas tipicamente internas são:

- operação de manutenção dos vários fatores produtivos;

- escolha e coordenação dos procedimentos de execução das várias operações empresariais;

- execução das várias operações produtivas para obtenção do produto. 
As atividades tipicamente externas são:

- aquisição de todos os materiais e de outros fatores produtivos necessários à produção;

- colocação dos produtos e subprodutos obtidos no mercado;

- operação de financiamento.

Comentando sobre as atividades internas à escolha do modo de execução das operações agrícolas e de manutenção dos fatores produtivos, Valle e Aloe (1981) afirmam que eles têm aspectos essencialmente técnicos, mas que, por suas repercussões influírem nas decisões administrativas, estas devem basear-se, em definitivo, em considerações de natureza econômica. Franco (1988) acrescenta que para obter os lucros na empresa rural predominam os aspectos de natureza econômica.

Sobre as atividades gerenciais, Crepaldi (1998) assegura que a tarefa de gerar informações gerenciais que permitam a tomada de decisão, com base em dados consistentes e reais, é uma dificuldade constante para os produtores rurais.

\section{INDICADORES DE DESEMPENHO}

Avaliar resultados e desempenhos faz parte do cotidiano. É da natureza humana. O indivíduo analisa seu desempenho no trabalho, o papel que exerce na família e na escola. Pela necessidade de ser reconhecido pelos seus sucessos, o ser humano está sempre se avaliando.

Uma empresa também necessita de um processo contínuo de avaliação de desempenho. Afinal, "o que não é medido não é gerenciado" (KAPLAN; NORTON, 1997, p. 21). É indispensável para uma empresa que pretende manter-se no mercado, na era da informação, controlar suas atividades operacionais, incentivar seus funcionários, identificar problemas que necessitam de intervenções dos gestores, feedback para orientar o planejamento, execução e controle, enfim, verificar se sua missão está sendo cumprida. Tudo isso pode ser feito por meio da avaliação de resultados e desempenhos.

Embora seja comum no cotidiano das empresas, avaliar resultados e desempenhos não é uma tarefa tão simples como pode parecer. Primeiro, é necessário estabelecer o que se pretende medir para, em seguida, estabelecer parâmetros adequados que avaliem o que se quer mensurar.

De acordo com Miranda; Wanderley; Meira (1999), enquanto a literatura tem mostrado que, no passado, as empresas tomavam decisões baseadas apenas em informações financeiras, obtidas da contabilidade das empresas, atualmente, as tomadas de decisão envolvem um maior número de variáveis, exigindo dos gestores uma grande preocupação com indicadores como: satisfação de clientes, qualidade dos produtos, participação no mercado, retenção de clientes, fidelidade dos clientes, inovação e habilidades estratégicas.

Banker et. al. (2000) consideram que as medidas não-financeiras são melhores indicadores de desempenho futuro do que as financeiras, auxiliando os administradores a focarem suas ações em perspectivas de longo prazo. Kaplan; Norton (1997) argumentam que os indicadores financeiros são inadequados para orientar e avaliar a trajetória da empresa em um ambiente competitivo, pois são indicadores de ocorrência, contam parte, mas não toda a história das ações passadas e não fornecem orientações adequadas para as ações que devem ser realizadas hoje e amanhã para criar valor financeiro futuro. Embora as medidas financeiras tenham sofrido críticas de estudiosos do assunto, não devem ser excluídas da avaliação de resultados e desempenhos, pois são provas concretas dos efeitos de todas as outras medidas. 
Os indicadores de desempenho são elementos fundamentais para a mensuração de performance, assim como para a definição das variáveis que melhor representem o desempenho geral de uma empresa.

Para cada setor de atividades, podem ser elaborados grupos distintos de indicadores de desempenho, obedecendo a suas características específicas, assim como a assimilação de conceitos metodológicos pelos profissionais responsáveis por sua elaboração.

A definição de indicadores de medida faz parte de uma seqüência lógica de procedimentos para desenvolvimento e implementação de um sistema de mensuração e avaliação de desempenho. Essas medidas devem ser orientadas para o futuro, procurando-se definir objetivos que traduzam as metas da organização.

Para Miranda; Silva (2002), os indicadores de desempenho desejáveis estão relacionados à definição das mensurações que de fato devem ser consideradas, nas quais devem ser identificados os atributos de desempenho relevantes que serão adotados como referência de avaliação.

Desse modo, torna-se possível a utilização de medidas de avaliação através de indicadores de eficácia, eficiência, qualidade, produtividade, inovação, custo e lucratividade.

Perez Jr.; Oliveira; Costa (2002) afirmam que a migração do ambiente tradicional de gestão e produção para os dias atuais deve ser acompanhada por profundas mudanças nos objetivos e nos conceitos referentes aos sistemas de avaliação de desempenhos individuais e da performance das unidades de negócios.

A utilização de indicadores de desempenho baseados em comportamentos padronizados não consegue identificar a origem de alterações de comportamento obtida através da melhoria da eficiência operacional. Dessa forma, aparentes ganhos de produtividade e eficiência podem decorrer de sazonalidades, tanto para insumos adquiridos no mercado interno em reais, quanto para insumos relacionados ao mercado externo, expostos às variações de preço em função da taxa de câmbio.

Aaker (2001) alega que uma das dificuldades na administração estratégica de mercado é o desenvolvimento de indicadores de desempenho que representem convincentemente as projeções de longo prazo.

Os sistemas tradicionais de mensuração de desempenho não são compatíveis com projeções de desempenho, pois a base de dados utilizada orienta uma análise financeira composta por valores históricos. Aspectos como lucratividade, participação de mercado, satisfação de clientes, prazos e incertezas dos fluxos de caixa futuros, dentre outros, não devem ser considerados como indicadores de tendência ou vetores de desempenho, pois expressam o desempenho após a ocorrência dos fatos.

Sobre a adoção de metodologias de mensuração de atividades produtivas, Bornia (2002) afirma que para simplificar o processo de controle de gestão devem ser consideradas medidas físicas de eficiência, eficácia e produtividade.

Os indicadores de mensuração de desempenho visam à mensuração da eficiência, mas é preciso lembrar que eles são apenas medidas de ocorrência.

\section{PROCEDIMENTOS METODOLÓGICOS}

Para a classificação da pesquisa, tomou-se como base a taxonomia desenvolvida por Vergara (2000), que a qualifica em relação a dois aspectos: quanto aos fins e quanto aos meios. Quanto aos fins, esta pesquisa é exploratória e descritiva. Exploratória porque procurou ampliar o conhecimento sobre indicadores de desempenho e descritiva porque buscou aplicar os indicadores de desempenho para comparar as unidades processadoras de mandioca localizadas nas sub-regiões do Estado do Ceará. 
Quanto aos meios, esta pesquisa é de campo e bibliográfica. Pesquisa de campo porque foi realizada nas unidades processadoras de raspas de mandioca do Estado do Ceará, onde foram coletados os dados. Bibliográfica porque foi realizado um estudo sistematizado, desenvolvido com base em materiais publicados: livros, revistas, jornais, internet, enfim, material acessível ao público em geral.

0 universo desta pesquisa é composto pelas unidades rurais processadoras de raspas de mandioca do Estado do Ceará, identificadas através do cadastro obtido junto ao Comitê Estadual da Mandioca (CMC), que forneceu todos os dados cadastrais das agroindústrias. Para Goode; Hatt (1979), pode-se recorrer a listas existentes no processo de definição do universo. Essa lista é composta por 158 unidades processadoras, localizados em 9 sub-regiões do Estado.

Para operacionalizar a disposição dos dados, foi considerada a localização geográfica das unidades processadoras das seguintes sub-regiões: Fortaleza, Baturité, Quixadá, Crato, Sobral, Crateús, Itapipoca, Ubajara e Limoeiro.

Os indicadores de desempenho foram agrupados obedecendo às variáveis relacionadas ao processo operacional da atividade-fim, bem como às variáveis vinculadas com as atividades comerciais. Os indicadores propostos para avaliar o desempenho operacional foram os seguintes:

- ganho financeiro sobre o preço:

$\Rightarrow$ esse indicador reflete a relação diferencial existente entre o preço pago pela matéria-prima adquirida junto aos associados e a matéria-prima adquirida junto a terceiros.

- fator de conversão da matéria-prima:

$\Rightarrow$ esse indicador reflete a relação existente entre o volume de matéria-prima processada e o volume de raspas produzidas.

Esses indicadores representam perspectivas distintas e independentes entre si, mas que são complementares para mensurar a performance interna das unidades processadoras. $O$ ganho financeiro sobre o preço agrega valor internalizado pela maior eficiência operacional dos associados em relação aos fornecedores externos e acarreta melhora na margem de lucro esperada. $O$ fator de conversão de matéria-prima representa a produtividade obtida em função da qualidade da matériaprima utilizada e contribui para ampliar economias de escala. Ou seja, maiores ganhos financeiros implicam maiores margens de contribuição, enquanto maiores fatores de conversão implicam menores custos fixos unitários.

Os indicadores propostos para ilustrar o desempenho econômico foram os seguintes:

- resultado operacional nominal:

$\Rightarrow$ esse indicador reflete a diferença nominal entre as receitas, as despesas e os custos totais.

- rentabilidade do negócio:

$\Rightarrow$ esse indicador reflete a participação relativa do lucro operacional obtido dentro do faturamento. 
Ambos indicadores representam perspectivas financeiras e econômicas, embora o resultado operacional tenha uma natureza nominal e a rentabilidade represente uma magnitude relativa. 0 resultado operacional nominal representa o lucro bruto obtido através das atividades econômicas e comerciais realizadas e pode ser influenciado pela escala de operações. A rentabilidade do negócio implica melhor eficiência econômica e financeira.

Os dados foram obtidos junto ao CMC e disponibilizados através de planilhas, as quais discriminavam as diversas variáveis estudadas em função das regiões nas quais as unidades processadoras estão localizadas.

Para atingir 0 objetivo proposto, utilizou-se 0 método de análise descritiva relativa aos indicadores investigados, visando identificar o desempenho dessas unidades processadoras. Para análise dos dados, utilizou-se o pacote estatístico SPSS - Statistical Package for the Social Sciences.

Cada indicador teve seus dados coletados a partir de valores nominais e foram convertidos para uma escala de 0 a 10, na qual o maior valor apresentado por uma unidade processadora tornouse equivalente a 10 e os demais foram calculados proporcionalmente.

Foram calculadas médias aritméticas para identificar o desempenho operacional e econômico das sub-regiões e, finalmente, uma média aritmética global de todos os escores.

\section{APRESENTAÇÃO E ANÁLISE SOS RESULTADOS}

Essa seção visa apresentar os principais aspectos que foram investigados junto aos indicadores propostos para avaliar o desempenho dessas unidades processadoras a partir dos referenciais propostos:

- ganho financeiro sobre o preço pago pelas matérias-primas;

- fator de conversão das matérias-primas;

- resultado operacional nominal e

- rentabilidade do negócio.

Inicialmente, investigou-se o ganho financeiro sobre o preço pago pela matéria-prima, cujos resultados são apresentados na Tabela 1.

Tabela 1 - Ganho financeiro sobre o preço pago pelas matérias-primas (R\$)

\begin{tabular}{|c|c|c|}
\hline Sub-Região & Valor Nominal & Escore \\
\hline Fortaleza & $-2,40$ & 07,58 \\
\hline Baturité & $-0,13$ & 09,65 \\
\hline Quixadá & 0,00 & 09,78 \\
\hline Crato & $-3,08$ & 06,70 \\
\hline Sobral & 0,22 & 10,00 \\
\hline Crateús & $-1,02$ & 08,76 \\
\hline Itapipoca & $-0,46$ & 09,33 \\
\hline Ubajara & $-2,76$ & 07,02 \\
\hline Limoeiro & 0,12 & 09,00 \\
\hline
\end{tabular}

Fonte: Pesquisa de Campo.

Nota 1: Os valores nominais estão expressos em Reais.

Nota 2: Os escores estão expressos em unidades. 
Considerando os dados apresentados na Tabela 1, constatou-se que apenas duas das subregiões estudadas registraram diferenças positivas entre os preços pagos a agricultores associados na compra de matérias-primas superiores aos preços pagos a terceiros. Esse resultado aponta uma fragilidade operacional bastante significativa, uma vez que o principal propósito da compra de matériasprimas produzidas pelos associados atuaria como uma atividade econômica complementar, responsável pela geração de receitas não operacionais complementares derivadas desse ganho.

Pode-se ainda afirmar que, até nas sub-regiões que registraram valores positivos (Quixadá, Sobral e Limoeiro), não foram encontrados valores positivos em magnitude significativa. Isso pode induzir a uma conclusão desfavorável a essa prática, uma vez que o esforço empreendido para a realização dessa atividade complementar não tem demonstrado uma efetiva materialização de ganhos financeiros encorajadores.

A razão pela qual essas diferenças não foram significativas reside na semelhança de padrões de produção apresentados entre os produtores associados e os produtores independentes. Ou seja, 0 estímulo à organização da produção, por meio de formação de grupos, não gerou curvas de aprendizagem que resultassem em maior eficiência sobre a produção.

Outro resultado relevante consiste nos valores encontrados para as sub-regiões de Fortaleza, Crato e Ubajara, que possuem as melhores condições climáticas e geológicas para a produção agrícola. Os valores obtidos apresentaram sinal negativo e foram significativos nominalmente. Nessas regiões, prevalece a maior eficiência dos produtores individuais, enquanto os associados focam suas atividades para as atividades de transformação.

A segunda dimensão de performance investigada foi o fator de conversão das matérias-primas. A Tabela 2 apresenta os resultados relativos à análise do fator de conversão das matérias-primas dentro do processo de transformação para cada uma das sub-regiões investigadas.

\section{Tabela 2 - Fator de conversão das matérias-primas.}

\begin{tabular}{lcc}
\hline \hline Sub-Região & Valor Nominal & Escore \\
\hline Fortaleza & 36,02 & 08,73 \\
Baturité & 36,06 & 08,74 \\
Quixadá & 41,22 & 10,00 \\
Crato & 38,43 & 09,32 \\
Sobral & 37,90 & 09,19 \\
Crateús & 39,16 & 09,50 \\
Itapipoca & 37,46 & 09,08 \\
Ubajara & 40,10 & 09,72 \\
Limoeiro & 34,50 & 08,37 \\
\hline \hline
\end{tabular}

Fonte: Pesquisa de Campo.

Nota 1: Os valores nominais estão expressos em percentuais.

Nota 2: Os escores estão expressos em unidades de escala variando entre 0 e 10.

Os resultados, obtidos através desse indicador de desempenho, sugerem um comportamento relativamente homogêneo, embora as sub-regiões de Quixadá e Ubajara possam se destacar ligeiramente por ter atingido patamares de conversão superiores a $40 \%$ no volume de matérias-primas processadas. Esse resultado indica que a qualidade das matérias-primas produzidas não apresenta discrepâncias significativas, mesmo considerando as diferenças de solo e densidade pluviométrica.

Tal característica pode ser visualizada a partir dos escores obtidos que variaram entre $10 \mathrm{e}$ 8,37 (apresentando uma amplitude inferior aos valores apresentados nos indicadores referentes aos ganhos financeiros sobre os preços pagos a associados, que variaram entre 10 e 7,02). Outro dado 
interessante consiste na proximidade observada nos fatores de conversão de seis das nove subregiões, que variaram entre $36,02 \%$ e $39,06 \%$. Essa relativa homogeneidade está respaldada pela difusão das práticas produtivas ao longo de toda a região, não se tratando de conhecimento exclusivo de determinados grupos.

A terceira dimensão de performance investigada foi o resultado operacional nominal. A Tabela 3 apresenta os resultados relativos à análise do indicador referente ao resultado operacional nominal para cada uma das sub-regiões investigadas.

Tabela 3 - Resultado operacional nominal.

\begin{tabular}{lcc}
\hline \hline Sub-Região & Valor Nominal & Escore \\
\hline Fortaleza & 221,25 & 0,56 \\
Baturité & $-110,68$ & 0,21 \\
Quixadá & 39,49 & 0,34 \\
Crato & $2.459,88$ & 2,92 \\
Sobral & $4.462,87$ & 3,97 \\
Crateús & 555,40 & 0,91 \\
Itapipoca & $9.182,34$ & 10,00 \\
Ubajara & $-316,13$ & 0,00 \\
Limoeiro & 370,55 & 0,72 \\
\hline \hline
\end{tabular}

Fonte: Pesquisa de Campo.

Nota 1: Os valores nominais estão expressos em Reais.

Nota 2: Os escores estão expressos em unidades de escala variando entre 0 e 10.

Os valores apresentados na Tabela 3 demonstram resultados operacionais divididos em dois grupos distintos. O primeiro grupo (composto pelas sub-regiões de Fortaleza, Quixadá, Crato, Sobral, Crateús, Itapipoca e Limoeiro) apresentou resultado operacional positivo, enquanto o segundo (formado por Baturité e Ubajara) caracterizou-se por resultados negativos ou quase nulos. Essa discrepância se materializa nos indicadores de desempenho calculados, que variaram de 0 a $10 \mathrm{em}$ função da enorme distância entre os valores máximo e mínimo encontrados.

Tabela 4 - Rentabilidade do negócio.

\begin{tabular}{lcc}
\hline \hline Sub-Região & Rentabilidade & Escore \\
\hline Fortaleza & 19,36 & 6,92 \\
Baturité & $-23,62$ & 0,00 \\
Quixadá & 25,76 & 7,95 \\
Crato & 13,44 & 5,97 \\
Sobral & 25,52 & 7,91 \\
Crateús & 38,45 & 10,00 \\
Itapipoca & 23,65 & 7,61 \\
Ubajara & $-9,65$ & 2,26 \\
Limoeiro & 33,21 & 9,15 \\
\hline \hline
\end{tabular}

Fonte: Pesquisa de Campo.

Nota 1: Os valores nominais estão expressos em percentuais sobre o faturamento.

Nota 2: Os escores estão expressos em unidades de escala variando entre 0 e 10. 
Faz-se necessário destacar que as sub-regiões de Crato, Sobral e Itapipoca caracterizam-se historicamente como grandes produtoras agrícolas. A principal razão para esses resultados está associada à escala de produção verificada nessas sub-regiões. A relativa concentração da produção e distribuição de matérias-primas foi decisiva para que elas se destacassem das demais.

Finalmente, foi considerada a rentabilidade do negócio. A Tabela 4 apresenta os resultados relativos à rentabilidade do negócio como indicador referente para cada uma das sub-regiões. Os resultados, obtidos por meio desse indicador de desempenho, mostram um comportamento relativamente distribuído, as sub-regiões de Crateús, Limoeiro, Quixadá e Itapipoca se destacam ligeiramente por terem atingido os maiores patamares de rentabilidade de negócio, chegando a $38,45 \%$.

É importante ressaltar que as sub-regiões de Crateús, Limoeiro e Quixadá apresentaram baixos resultados operacionais nominais (respectivamente $R \$ 555,40, R \$ 370,55$ e $R \$ 39,49$ ). Por outro lado, as sub-regiões de Baturité e Ubajara apresentaram valores negativos, o que representa uma efetiva redução do negócio nessas sub-regiões.

Excluindo essas duas sub-regiões, os valores dos escores obtidos variaram entre $10 \%$ e 5,97\% (apresentando uma amplitude inferior aos valores apresentados nos indicadores referentes ao resultado operacional nominal que, excluindo Itapipoca, variou entre 3,97\% e 0,00\%). Esse resultado vai ao encontro dos valores obtidos através do fator de conversão de matérias-primas que também possui características que expressam valores relativos.

Após identificar os resultados individuais inerentes a cada uma das dimensões de performance investigadas, foram calculados escores representativos para a perspectiva operacional e para a perspectiva econômica. A Tabela 5 apresenta os resultados relativos aos escores médios parciais para as variáveis operacionais e para as variáveis econômicas.

\section{Tabela 5 - Escores médios parciais.}

\begin{tabular}{lcc}
\hline \hline Sub-Região & Escore Operacional & Escore Econômico \\
\hline Fortaleza & 8,15 & 3,74 \\
Baturité & 9,19 & 0,10 \\
Quixadá & 9,89 & 4,14 \\
Crato & 8,01 & 4,44 \\
Sobral & 9,59 & 5,94 \\
Crateús & 9,13 & 5,45 \\
Itapipoca & 9,20 & 8,80 \\
Ubajara & 8,37 & 1,13 \\
Limoeiro & 8,68 & 4,93 \\
\hline \hline
\end{tabular}

Fonte: Pesquisa de Campo.

Nota 1: Os escores médios estão expressos em unidades de escala variando entre 0 e 10.

Os resultados, obtidos através desses indicadores de desempenho, mostram um comportamento relativamente homogêneo para as variáveis relacionadas às características operacionais em comparação com os valores apresentados pelos escores relativos aos fatores econômicos estudados.

Desse modo, pode-se afirmar que as sub-regiões apresentam um perfil de desempenho operacional semelhante, no qual os escores médios para suas respectivas variáveis variaram entre 8,01 e 9,59 (Crato e Sobral, respectivamente) em comparação aos escores médios de desempenho econômico, que variaram entre 0,10 e 8,80 (Baturité e Itapipoca, respectivamente). 
Por outro lado, o escore econômico da sub-região de Itapipoca se destaca dentre os demais. Esse resultado foi fortemente influenciado por seu resultado operacional nominal, muito superior aos demais em função do elevado volume da escala de operações da referida sub-região.

Para dar uma visão mais genérica sobre as dimensões de mensuração de desempenho investigadas, foi calculado um escore geral de desempenho. A Tabela 6 apresenta os resultados relativos aos escores médios gerais, abrangendo os escores relativos aos indicadores de desempenho operacionais e econômicos.

Tabela 6 - Escores médios gerais.

\begin{tabular}{lc}
\hline \hline Sub-Região & Escore Médio geral \\
\hline Fortaleza & 5,94 \\
Baturité & 4,65 \\
Quixadá & 7,01 \\
Crato & 6,22 \\
Sobral & 7,76 \\
Crateús & 7,29 \\
Itapipoca & 9,00 \\
Ubajara & 4,75 \\
Limoeiro & 6,81 \\
\hline \hline
\end{tabular}

Fonte: Pesquisa de Campo.

Nota 1: Os médios gerais escores estão expressos em unidades de escala variando entre 0 e 10.

Os resultados, obtidos através dos escores médios gerais derivados dos indicadores de desempenho, demonstram um comportamento relativamente determinado pelos escores econômicos, principalmente pelo resultado operacional nominal, uma vez que estes apresentaram maior amplitude de valores em comparação aos escores operacionais.

Faz-se interessante observar que as sub-regiões de Baturité, Ubajara e Fortaleza obtiveram os três piores escores de desempenho econômico $(0,10,1,13$ e 3,74, respectivamente), apresentando, também, os três piores escores médios gerais $(4,65,4,75$ e 5,94, respectivamente). Essas sub-regiões não possuem tradição agrícola, portanto esse resultado não chega a ser surpreendente.

Por outro lado, as sub-regiões de Itapipoca e Sobral, que haviam obtido os melhores escores de desempenho econômico ( 8,80 e 5,94, respectivamente), também apresentaram os dois melhores escores médios gerais $(9,00$ e 7,76 , respectivamente).

\section{CONCLUSÕES}

Considerando as informações apresentadas ao longo da discussão comparativa acerca dos dados relativos aos escores de desempenho propostos para comparar as diversas sub-regiões, as conclusões foram organizadas obedecendo à mesma disposição, buscando facilitar a identificação das semelhanças e diferenças entre as variáveis analisadas.

O fortalecimento da capacidade organizacional dos grupos de produtores é um objetivo a ser atingido pelo projeto integrado, embora os resultados obtidos ainda não sejam suficientes para dar suporte a essa melhora. 
Outro aspecto relevante está associado ao êxito no fornecimento adequado de matérias-primas para as unidades de processamento, que se tornou o principal desafio sobre a eficiência e o desempenho organizacional das associações.

Saber definir o que deve ser medido e avaliado nas diferentes atividades realizadas por uma empresa não é uma tarefa simples. Determinar quais as medidas que devem ser realizadas depende da complexidade do processo que se deseja avaliar, da sua importância em relação às metas estabelecidas pela empresa e da expectativa de uso gerencial posterior desses dados.

Sobre os indicadores de desempenho relacionados aos aspectos operacionais, os resultados demonstraram comportamentos relativamente semelhantes entre as diversas sub-regiões, sem a identificação de extremos significativos. Tanto para os ganhos financeiros obtidos pelos preços pagos pelas matérias-primas, quanto para os fatores de conversão das matérias-primas, os resultados obtidos não revelaram desempenhos discrepantes.

No que se refere aos indicadores de desempenho relacionados às variáveis econômicas, podese afirmar que as sub-regiões de Crato, Sobral e, principalmente, Itapipoca apresentam um perfil de desempenho operacional superior às demais, que foi determinado pela escala econômica das atividades. Quanto à rentabilidade do negócio, os resultados mostraram comportamentos relativamente semelhantes entre as diversas sub-regiões, sem a identificação de extremos significativos.

Os resultados encontrados através da estimação dos escores operacionais médios confirmaram a ausência de discrepância entre os valores obtidos pelas diversas sub-regiões investigadas. No entanto, os escores econômicos médios revelaram que a sub-região de Itapipoca se destacou das demais, influenciada, principalmente, pelo indicador referente ao resultado operacional nominal. Os resultados apresentados pelos escores médios gerais apontam que as sub-regiões de Itapipoca e Sobral apresentam os maiores escores. Essas duas sub-regiões também apresentaram os maiores escores referentes ao resultado operacional nominal.

Portanto, as diferenças encontradas entre o desempenho das diversas sub-regiões investigadas residem, principalmente, na escala de operações. Pois os resultados referentes às demais dimensões de desempenho investigadas (ganhos sobre o preço das matérias-primas, taxas de conversão das matérias-primas e rentabilidade do negócio) não influenciaram os escores médios gerais com a mesma intensidade, nem conseguiram anular sua discrepância.

Investigações sobre a mensuração de desempenho no âmbito do agronegócio são difíceis de ser implementadas, bem como as referências bibliográficas publicadas sobre essa temática são escassas. Sendo assim, estudos mais aprofundados e mais abrangentes são necessários para poder lançar luzes sobre esse setor econômico que é de fundamental importância para o desenvolvimento da economia brasileira. Além disso, a utilização de indicadores de desempenho possui um papel relevante na gestão de empresas agroindustriais inseridas no atual contexto global competitivo.

\section{REFERÊNCIAS}

AAKER, David A. Administração Estratégica de Mercado. 5. ed. Porto Alegre: Bookman; 2001.

BANKER, Rajiv D.; POTTER, Gordon; SRINIVASAN, Dhinu. An empirical investigation of an incentive plan that includes nonfinancial performance measures. The Accounting Review, Florida, v.75, n.1, p.65-92, jan.2000.

BORNIA, Antonio Cezar. Análise gerencial de Custos. Porto Alegre: Bookman; 2002.

CREPALDI, Silvio. Contabilidade Rural. 2. ed. São Paulo: Atlas; 1998. 
GOODE, Willian J. HATT, Paull K.K. Métodos em Pesquisa Social. 7. ed. São Paulo: Atlas; 1979.

JANK, Marcos Sawaya; NASSAR, André Meloni. Competitividade e Globalização. Economia e gestão dos negócios agroalimentares (Décio Zylbersztajn e Marcos Fava Neves -ORG). São Paulo: Pioneira; 2001.

KAPLAN, Robert S.; NORTON, David P. A estratégia em ação: balanced scorecard. Tradução: Luiz Euclydes Trindade Frazão Filho. 5. ed. Rio de Janeiro: Campus, 1997.

MARION, José Carlos. Contabilidade Rural. 6. ed. São Paulo: Atlas; 2000.

MARION, José Carlos; SANTOS, Gilberto José dos; SEGATTI, Sonia. Administração de Custos na Agropecuária. 3. ed. São Paulo: Atlas, 2002.

MATTOS, Zilda Paes de Barros. Contabilidade Financeira Rural. São Paulo: Atlas; 1999.

MAZZALI, Leonel. 0 processo recente de reorganização agroindustrial: do complexo à organização em rede. São Paulo: UNESP, 2000.

MEGIDO, José Luiz Tejon; XAVIER, Coriolano. Marketing e Agribusiness. 4. ed. São Paulo: Atlas; 2003.

MINISTÉRIO DA AGRICULTURA, PECUÁRIA E ABASTECIMENTO. Disponível em http://www.agricultura.gov.br. Acesso em 07 de Março de 2005.

MIRANDA, L. C.; WANDERLEY, C.A.; MEIRA, J.M. Garimpando na imprensa especializada: uma metodologia alternativa para a coleta de indicadores de desempenho gerencial. IN: CONGRESSO INTERNACIONAL DE CUSTOS, VI. Anais ... Portugal, 1999.

MIRANDA, Luiz Carlos; SILVA, José Dionísio Gomes da. Medição de desempenho. In SCHMIDT (ORG) Controladoria. Porto Alegre: Bookman; 2002.

PEREZ JR, José Hernandez; OLIVEIRA, Luis Martins de; COSTA, Rogério Guedes. Gestão Estratégica de Custos. São Paulo: Atlas; 1999.

VALLE, Francisco. Manual de Contabilidade Agrária. São Paulo: Atlas; 1985.

VALLE, Francisco; ALOE, Armando. Contabilidade Agrícola. 7. ed. São Paulo: Atlas; 1981.

VERGARA, Sylvia Constant. Projetos e relatórios de pesquisa em administração. 3. ed. São Paulo: Atlas, 2000.

\title{
ECONOMIC AND OPERATIONAL PERFORMANCE MEASUREMENT: A EXPLORATORY STUDY IN AGRIBUSINESS CONTEXT.
}

\begin{abstract}
Considering the relevance of performance measurement to corporate management, this study has the objective to elaborate and apply some performance measurement tools to compare rural organizations located in Ceará. In this research, 158 rural production units from 9 regions o were measured. The performance indicators were developed considering the operational issues, as well as some variables related to commercial activities.
\end{abstract}

Key words: Performance measurement. Agribusiness. 